\title{
Revisiting the Perception of Silence in Linguistics
}

\author{
Said al Jahdhami \\ Department of English Language and Literature, College of Arts and Social Sciences, Sultan Qaboos \\ University, P.O. Box 42, P.C. 123, Muscat, Oman \\ saidj@squ.edu.om
}

\begin{abstract}
Silence in human communication has not only been marginalized as a means of communication, but has often been considered as void of serving any communicative role. It is presumptuously viewed as the mere opposite of communication occurring at the far end of the communicative continuum. Investigating the role of silence in human communication from a linguistic point of view, this paper, hence, attempts to show that such conventional view of silence as a non-communicative tool should be reconsidered. It argues that both silence and speech make an integral part of human communication as they coincide in any delivered message. The context in which they serve a communicative function plays a major role in their use, the choice of one over the other, as well as the interpretation of any delivered message. The study also argues that speech and silence do not always fall into the traditional schematic classification of vocal versus non-vocal. While silence can be vocal in some occasions in human communication, speech can be expressed non-vocally too.
\end{abstract}

Key Words: Silence, Speech, Communication, Vocal, Non-vocal

\section{Introduction}

Speech and silence have often been erroneously viewed as having two dichotomous exclusive functions. Speech is maintained to express language and avoid disrupting communication. Silence, on the other hand, is largely applied to halt the flow of expressing language and thus break down communication (Jaworski, 1993; Eggins \& Slade, 1997; Zou, 2002; Voegelin, 2010). Accordingly, speech is regarded as the mere opposite of the absence of sounds, namely those ones used to deliver semantic functions. Likewise, silence is considered as a separate entity from speech assuming that silence, contrary to speech, is devoid of linguistic meaning that could be expressed via means other than verbal ones. This view also entails that meaning can only be expressed via vocalization, which, hence, undermines any role of silence in human communication. This view, however, appears to be erroneous, for both speech and silence can be applied to express language and keep channel of communication stream between the sender and the receiver of a given message. As a matter of fact, the presence of sounds (i.e. speech) does not necessarily entail communication; sounds can be meaningless if they make no sense or if they are unintelligible to the listener. By the same token, the absence of sound does not necessarily suggest absence of communication. Unlike speech which requires one's knowledge of a given language, silence is a universal language that could indeed express meanings intelligible to all humans irrespective of their linguistic background. Cases in point, for instance, are moments of farewell and quarrel. Silence in such situations is more expressive than speech for it is applied to avoid sensual moments or confrontation of discussion respectively. Just as humans have the capability of palavering a long talk communicating nothing expressive, they have the capability of expressing meaningful messages without applying the minimum level of verbosity. This suggests that there can be meaningful silence and meaningless speech because it is the conveying of a meaningful message what counts in communication rather than the absence or presence of sounds per se.

\section{Literature Review}

A look into the literature on silence shows that silence has often been defined as the absence of speech or time at which there is no speech or vocalization to be heard, which gives an audacious view that hearing no sound corresponds to silence (Jaworski, 1993; Eggins \& Slade, 1997; Zou, 2002,Voegelin, 2010). Nudds (2001), however, 
argues that both speech and silence can be heard. Hearing the latter should be defined as a successful perception of absence of sounds rather than failure to hear sound or meanings provided by such absence of sounds. This suggests that silence does involve the presence of meanings, but expressed via a non-verbal means rather than verbal ones ordinarily used in human communication. We, as humans, are geared up to recognize 'silence' and 'absence of silence' but recognizing silence is not enough to hear silence. A deaf person can recognize silence due to the failure to hear sound, but cannot hear silence as the presence and absence of sounds are identical to him. A hearing person, on the other hand, can recognize and hear the absence of sound employed for a particular purpose. Such silence is referred to as 'eloquent silence' or 'communicative silence' as opposed to non-communicative silence. It is the type of silence that is deliberately chosen by the speaker to communicate a message (Ephratt, 2008).

Eloquent silence is not uncommon in human communication due to the several communicative functions it could serve. From a discourse function point of view, it is argued that silence, just like speech, can be used to perform several linguistic functions such as excalmatives, imperatives, decleratives and interrogatives (Jaworski,1993 \& Bruneau ,2008). Politeness theory stipulates that silence could be used to avoid face threatening acts (FTA) that risk face whereby silence is applied to protect 'self-image' (Mao, 1994). Showing solidarity and rapport with the addressee as well as marking turn taking between the interlocutors to prevent one from taking over another's turn are other functions of silence (Zuo, 2002). Bruneau (2008) states that silence could have pausal, junctural and paralinguistic features, all of which are meaningful silences utilized to demarcate utterances. Silence could also be used to give feedback in addition to other means such as head nods, facial expressions and direction of gaze and minimal responses such as mhmm, ooh and yeah (Eggins \& Slade, 1997). Zuo (2002) distinguishes between different functions of silence: prosodic (used to mark word boundaries, punctuation, pronunciation and emphasis), cognitive (related to syntactic and semantic planning and choice of word), stylistic (reflecting conversational styles), and social interactive and communicative silences (such as unwillingness to converse about a topic, showing agreement ..etc.).

Several reasons have been cited to be the causes of silence in human communication. Berger (2004) highlighted three causes of silence: unexpected information/deviant behavior, extreme emotions and lack of information or knowledge. Kurzon (1995) makes a distinction between intentional and unintentional silence; the former has an internal source triggered by the speakers own will whereas the latter is imposed by an external source. Kurzon also distinguishes between conversational (explicit) and thematic (implicit) silence whereby the former is refusing to make utterances in a conversation while the latter is choosing not to talk about a particular topic. Linguists also draw attention to broad line cases that can fall between the two extremes such as memory failure where one does not really find what to say and is partially forced to remain silent. The same holds true for hesitation where both silence and speech seem to overlap in the speaker's production of language, which gives evidence to the robust connection between silence and speech in human communication (Kurzon ,1995, Eggins \& Slade, 1997).

Ephratt (2008) draws the difference between silencing and eloquent silence; the former involves the power of someone/something over the speaker to be silent whereas the latter is chosen voluntarily by the speaker to communicate a message via silence. Semantically, Arabic distinguishes between two words that could possibly be used to refer to silence: 'sukoot' and 'summt'. 'Sukoot' is silence after talk that is usually made out of one's own will. It is shorter in duration and it is a type of negative silence since it is refraining from expressing something true or false. 'Summt', on the other hand, is no talk at all and is usually by one's own will and is longer in duration than 'sukoot'. It is regarded as positive silence since it is refraining from expressing something foolish, trivial or untrue. Silence of a suspect who refused to admit his wrong doing before the judge and silence used as means to express feelings in a funeral, in religious ceremonies or in prayer are two examples that juxtapose 'sukoot' and 'summt', respectively.

It should be noted that since humans are likely to misinterpret speech, they are more likely to misinterpret silence owing to the fact that silence is culture dependent. Its interpretation, thus, is subject to several variables such as beliefs, gender, speakers and personal speaking style (Nakane, 2006). Eggins \& Slade (1997) state that 
silence can be salient in some cultures and genders with some viewing avoiding silence as being a cooperative conversational partner while others give emphasis to silence and indirect feedback in a conversation. For instance, taciturnity is positively viewed in some Asian communities whereas it is viewed negatively in America since it symbolizes death and is viewed as a sign of a boring conversational partner. This, in turn, creates awkward situations in interaction between Americans and Asian people as each would have a different view in the interpretation of silence (Bruneau, 2008). Compared to students from Britain and those from other cultures, Wong (2003) found that silence has more significant role in Japanese students' conversation that the other groups including the British group who tend to apply less visual and non-verbal communication means compared to Japanese students. Silence in the Japanese culture appears to perform different tasks such as showing a sign of refusal, disagreement and rejection expressed in a polite manner so that violating the record of politeness is avoided. In an Indian culture, for instance, Barltelt (2010) establishes that American Indian students would regard responding to a teacher's question as a rude act that set one as above other peers. Students, instead, would remain silent and avoid eye contact with the teacher to communicate respect and politeness to their teacher. Such acts to instructors from occidental cultures, however, are viewed as showing mere impoliteness to the teacher. Findings of this sort show that the interpretation of silence should not be deracinated from its cultural context, for silence is largely culture specific serving different functions in different cultures.

\section{The Interconnectivity of Silence and Speech}

It has been attested that language can be expressed via verbal and non-verbal means alike, both of which can be used to express several linguistic functions (Jaworski, 1997; Kurzon, 2007 ; Ephratt, 2008 \& Ephratt, 2011). Humans tend to make use of both verbal and non-verbal means in communication with one means more prominent than the other in some cases depending on the context in which communication takes place. It can be argued, however, that both speech and silence make an essential part of any single message exchanged between two different beings in human communication. It is imperative that both components (speech and silence) coexist in any particular message so that it is successfully delivered, with each element taking place at a particular point in the course of delivering that message. Adopting the linguistic term from Phonology, both silence and speech seem to largely occur at 'complementary distribution' in a particular conversation where one does not occur in the position of the other, but both complement one another and make an integral input to communicating a given message. The term 'complementary' suggests that both speech and silence coincide in a given conversation as both are required to team up for the making of a given conversation/ message. Speech and silence, however, do not befall into a vacuum. Since a linguistic message is delivered within a context, it plays a chief role in our choice of which means (speech or silence) should take place at a particular point in a conversation as well as which means is more salient and effective than the other in delivering a particular meaning. Context as used here corresponds to the referential function in Jakobson's communicative model (1960). It refers to the outside world that is dependent and external in reference to both the speaker (i.e. the emotive function) as well as the addressee (i.e. the conative function). It is the setting in which a message is exchanged between a speaker and an addressee which significantly necessitates the presence of both speech and silence so that a message is conveyed. When a conversation takes place between two interlocutors, both speech and silence are existent concurrently within the same context; the speaker speaks (speech is involved) and the addressee listens (silence is involved) at the same time. The speech component, however, is activated by the speaker and the silence component is activated by the addressee. The speaker is to activate speech to deliver a message, and the addressee is to activate silence to be able to process the speakers' message. Speech of one party (i.e. speaker) communicates the creation of a message delivered to another party (i.e. addressee). Silence of the addressee, on the other hand, communicates the willingness to receive that message and to respond to it appropriately. Silence of the speaker at the end of an utterance is required to demarcate utterances and to give a sign for turn taking whereby silence is activated by the ex-speaker (the newly allocated addressee) and speech is activated by the ex- addressee (the newly allocated speaker). Swapping the speaker- addressee roles indicates the presence of both speech and silence within the same context in a particular conversation as exemplified underneath. 

A (speaker): speech is activated.
B (addressee): silence is activated.
B (speaker): speech is activated.
$\rightarrow \quad$ A (addressee): silence is activated.

By the same token, when a reader reads a text, the speech component is activated by the writer, and simultaneously the silence component is activated by the reader. When the reader comes to a halt to think about the text and probably ponders some questions in mind (speech), turn taking is involved. The writer is silent here though his silence is intentionally made by the reader's will who chooses to put the writer to silence by pausing reading. The reader comes to speech though the writer is not listening to the speech of the reader due to his physical absence. It is noteworthy, however, that speech and silence do not always follow one another in a mechanical manner in human communication. They can be activated at the same exact moment by both parties involved in human communication; speech of two interlocutors may overlap (i.e. a couple arguing over whose mistake was losing their savings), or their silence may overlap too (i.e. the same couple remaining irritably silent trying to figure out a way-out of such predicament). There is communication in both situations but via different means, which shows that speech and silence seem to co-exist in our communication to serve a communicative function whether they follow one another or co-occur at the same very moment.

Noteworthy is that speech and silence, as means of communication, are both within humans' disposal, and fascinatingly may substitute one another. Their choice, however, is subject to our judgment of which element is better effective in a particular situation. In other words, where one (speech or silence) fails to express the intended meaning, it is subject to leave the way for the other to intervene and better express the intended meaning. This corresponds to what Rescher (1998) calls 'filling the gap' in doing the communicative work. Rescher argues that means of verbal communication cannot always be within speakers' disposal to be explicit in speech, and thus silence (non-verbal means of communication) is complementary to fill the gap to do the communicative work. We, as humans, are likely to recognize the best element to express the intended meaning irrespective of its verbal or non-verbal nature. Consider the following conversation.

A: How was the job interview? Did you get the job?

\section{B: Silence}

\section{A: Why? You make a perfect candidate. What went wrong?}

The silence of speaker B was simply vocalizing the answer 'No, I did not get the job because I did not pass the interview.' which made speaker A responds to such non-verbal message with a verbal means. It is an unmarked answer stated through silence rather than speech. Speaker B could have responded using verbal means, but it seems that it suffices to give an eloquent unmarked answer via silence rather than giving a verbal answer so that the intended meaning gets across efficiently.

Likewise, in answering a rhetorical question, for example, silence is more prominent than speech. It is a question whose answer is conveyed with eloquent silence rather than the common vocal answer. God's question in the Holly Quran addressed to his servants who do not believe in his ability to resurrect them "Is not God able to resurrect the dead?" ends the chapter with an unmarked answer that echoes in the reader's mind. This silence states via a referential answer that God is unquestionably able to resurrect the dead. God does not seek a verbal answer; the answer is rather expressed via eloquent silence where such silence admits the answer to such question. On the other hand, an answer to a question like 'How can I get to the hospital?' cannot be via an unmarked answer through silence unless it is meant to communicate anger or unwillingness to communicate with the speaker. It rather requires speech that shows the way to be followed to arrive at that particular hospital.

Notable is that our speech and silence, as humans, tend to encompass both stated and unstated meanings simultaneously. Expressing some meanings via speech may comprise articulating other unstated meanings. Similarly, expressing some meanings via silence may comprise stating other understood meanings. In other words, we tend to express both explicit and implicit meanings in our conversations. This goes in line with the 
referential function in Jakobson's communicative model (1960) which entails that omitting something (i.e. silence) is equal to stating something (i.e. speech). An answer like '/ speak French only' to a question like "What Romance languages do you speak?" gives referential meaning to the unsaid meaning '/ speak French, but I do not speak Spanish, Italian or any other Romance language". One who is asked about the number of his kids and answers that he is bachelor gives referential meaning to the unsaid meaning that he has no kids and he is not married in the first place. Similarly, a student's silent answer to an instructor's question 'have you spent your weekend doing your work?' gives referential meaning to the unsaid meaning 'I spent my weekend doing something else other than doing my work'. A parent's silent answer to a kid's question 'are we going into a picnic this weekend?' gives referential meaning to the unsaid meaning 'we are staying home this weekend'. Indeed we, as humans, are primed to understand both the stated and unstated meanings in our conversations though we tend to focus on the stated ones.

\section{Vocal vs. Non-Vocal Classification}

Categorically, speech and silence have been considered as the two end points of the communicative continuum; the former has often been characterized as vocal whereas the latter has been characterized as non-vocal (Jaworski, 1997; Ephratt, 2011). This view is true to some extent. It can be argued, however, that speech and silence should not be strictly classified into such an inert schema of vocal versus non-vocal classification. The opposite, in fact, is equally true as silence proves to be vocal in some cases, and as non-verbal means can be used to express speech. Human language exhibits several examples of vocalization via silence. One example of such vocalization is the zero sign in Morphology. Zero sign does not express something vocally, but it functions as a vocal symbol since it is used as a numerical character just like the plural marker 's'. The following examples juxtapose plurality vocalized by the silent zero sing as opposed to plurality vocalized by non-silent plural 's'.

one table vs. two tables (non-silence) one fish vs. three fish- $\varnothing$ (vocal silence).

one book vs. few bookS (non-silence) one sheep vs. several sheep- $\varnothing$ (vocal silence).

Silence (i.e. zero sign) in such cases is vocal since it does express plurality as opposed to plurality expressed by applying the explicit plural 's' or singularity expressed via the absence of 's'. Likewise, the zero sign of the past tense marker of some English verbs, such as those exemplified underneath, represent vocal silence. The zero sign in such verbs vocalizes the (past) tense of these verbs via silence as opposed to the -ed suffix used in regular verbs or the third person singular 's' marking present tense, as illustrated in the beneath examples.

\section{Ahmed cutS (non-silence) wood. $\quad$ vs. Ahmed cut- $\varnothing$ (vocal silence) wood.}

Khalil putS (non-silence) his books there vs. Khalil put- $\varnothing$ (vocal silence) his books there.

The same holds true for the passive voice. When the agent/doer of an action is unknown, its occurrence is of no use, or if focus is meant to be more on the object, it is expressed via referential silence that makes its presence redundant. Although the passive voice excludes the doer syntactically, it vocalizes it semantically via making referential meaning to it. It shifts focus from the 'agent' argument of the verb (i.e. subject) to the 'patient' argument of the verb (i.e. object) which results in fronting the latter and omitting the former. The optionality of mentioning the doer via using the standard 'by $X$ ' where $X$ is the doer (ex. The criminal was caught 'by the police'.) gives evidence that the doer is vocalized in the passive voice structure but rather by using silent signs. The context in which such silent signs occur plays a role in their vocalization suggesting that their absence is meant to serve a function; it contributes a meaningful vocal silence.

By the same token, speech can be non-vocal as it can be communicated non-vocally in human communication. A concrete example of such fact is sign language used in human communication whereby not much value is given to the presence of sounds in conveying meanings. Communication in sign language is attained via the 'absence of sounds' (i.e. signing) which signifies 'silence' in spoken language. Silence in sign language is attained via bringing signing to a stop used either to demarcate the end of a delivered message and thus show turn 
taking, or to deliver a message via silence (non-signing), just like delivering a message through silence in spoken language. As a matter of fact, 'speech' in spoken language corresponds to 'signing' in sign language whereas 'silence' in spoken language corresponds to 'non-signing' in sign language, all four of which are employed as means of communication in human language.

$$
\begin{array}{llc}
\text { Spoken language } & \text { vs. } & \text { Sign language } \\
\text { Speech (presence of sounds) } & \rightarrow & \text { signing /non-vocalized speech (presence of signs) } \\
\text { Silence (absence of sounds) } & \rightarrow & \text { non-signing/ eloquent silence (absence of signs) }
\end{array}
$$

Just as speakers are primed to recognize and hear eloquent silence (absence of sounds) employed to deliver an expressive message, singers too can recognize and hear eloquent silence (absence of signs) used to deliver a meaningful message. Sign language, in fact, gives ample evidence that speech is not necessarily delivered through vocalization. Signers still exchange messages and communicate with one another via active use of hands and head movement without employing a single sound. Two speakers au fait with sign language can deliver the same exact message either by using vocalization (presence of sounds) or signing (absence of sounds). Whether they opt for a 'vocalized' or 'non-vocalized' speech still there is a message communicated. The same two communicators may communicate with one using sounds (vocalized speech) while the other using signs (non-vocalized speech) in the same conversation. Indeed humans are so fortunate that speech can be delivered by means other than verbal noes so that such 'non-vocalized' speech (sign language) enables (hearing) speakers to deliver speech to deaf/dumb people and equally comprehend speech non-vocalized by signers.

\section{Conclusion}

The paper advanced the claim that although speech and silence are often perceived as means for expressing language versus breaking down communication, both can be used to serve significant communicative roles. Silence, just like speech, can be utilized to express meaningful messages, but via non-verbal means as opposed to the widely used verbal means (i.e. speech). Speech and silence exist simultaneously in a conversation/message, and both contribute fundamentally to the well-delivery of a message. As means of communication, they are both within humans' disposal. The context in which they occur, however, plays a major role in determining which one is more operative in delivering a given message as well as which one should be prominent at a certain point. Likewise, the presence and absence of sounds does not seem to exclusively categorize speech and silence in human communication. Just as silence could serve a vocal function, speech can be expressed non-vocally in human communication.

\section{References}

1. Barltelt, G. (2010). American Indian silence in House made of dawn. California Linguistic Notes, 1, 1-20

2. Berger, C, R. (2004). Speechlessness: causal attributions, emotional features and social consequences. Journal of Language and Social Psychology 23 (2), 147-179.

3. Bruneau, T. J. (2008). How Americans use silence and silences to communicate. China Media Research, 4(2), 77-85

4. Eggins, S. (2004). An Introduction to Systemic Functional Linguistics. London, UK: Continuum

5. Eggins, S., \& Slade, D. (1997). Analyzing Casual Conversation. London: equinox.

6. Ephratt, M. (2008). The functions of silence. Journal of Pragmatics 40, 1909-1938 
7. Ephratt, M. (2011). Linguistic, paralinguistic and extralinguistic speech and silence. Journal of Pragmatics 43, 2286-2307

8. Jakobson, R . (1960). Concluding statement: linguistics and poetics. In: Sebeok, Thomas A. (Ed.), Style in language. Wiley, New York, pp. 350-377.

9. Jaworski, A. (1993). The Power of Silence: Social and Pragmatic Perspectives. Sage, Newbury Park, CA.

10. Jaworski, A (Ed.). (1997). Silence: Interdisciplinary Perspectives. Berlin: Mouton de Gruyter.

11. Kurzon. D. (1995). The right of silence: A Socio-pragmatic model of interpretation. Journal of Pragmatics, 23,55-69

12. Kurzon. D. (2007). Towards a typology of silence. Journal of Pragmatics, 39,1673-1688

13. Mao, L. R. (1994). Beyond politeness theory: 'Face' revisited and renewed. Journal of Pragmatics, 21 (5), 451586

14. Nakane, I. (2006). Silence and politeness in intercultural communication in

15. university seminars. Journal of Pragmatics, 38 1811-1835

16. Nudds, M. (2001). 'Experiencing the Production of Sounds'. European Journal of

17. Philosophy, 9, 210-99.

18. Rescher, N. (1998). The significance of silence. European Review 6 (1), 91-95.

19. Voegelin, S. (2010). Listening to Noise and Silence: Towards a Philosophy of Sound Art. New York: USA

20. Wong, N. L. (2003). The communicative functions and meanings of silence: An analysis of cross cultural views. Journal of TagenBunka, 3, 125-146

21. Zuo, Y. (2002). The Golden Silence: Pragmatic Study on Silence in Dyadic English Conversation. Muenchen: LINCOM Europa. 